\title{
Zerumbone Restores Gut Microbiota Composition in ETBF Colonized AOM/DSS Mice
}

\author{
Hye-Won Cho ${ }^{1}$, Ki-Jong Rhee ${ }^{2}$, and Yong-Bin Eom ${ }^{1,3 *}$ \\ 'Department of Medical Sciences, College of Medical Sciences, Soonchunhyang University, Asan 31538, Republic of Korea \\ ${ }^{2}$ Department of Biomedical Laboratory Science, College of Health Sciences, Yonsei University at Wonju, Wonju, \\ 26493, Republic of Korea \\ ${ }^{3}$ Department of Biomedical Laboratory Science, College of Medical Sciences, Soonchunhyang University, Asan \\ 31538, Republic of Korea
}

Colorectal cancer (CRC) is the leading cause of common malignant neoplasm worldwide. Many studies have analyzed compositions of gut microbiota associated with various diseases such as inflammatory bowel diseases (IBD) and colon cancer. One of the most representative bacteria involved in CRC is enterotoxigenic Bacteroides fragilis (ETBF), a species belonging to phylum Bacteroidetes. We used ETBF colonized mice with azoxymethane (AOM)/dextran sulphate sodium (DSS) and zerumbone, a compound with anti-bacterial effect, to determine whether zerumbone could restore intestinal microbiota composition. Four experimental groups of mice were used: sham, ETBF colonized AOM/DSS group, ETBF colonized AOM/DSS group zerumbone $60 \mathrm{mg} \mathrm{kg}^{-1}$ (ETBF/AOM/ DSS + Z (60)), and only zerumbone (60 $\left.\mathrm{mg} \mathrm{kg}^{-1}\right)$-treated group. We performed reversible dye terminators-based analysis of 16S rRNA gene region V3-V4 for group comparison. Microbiota compositions of ETBF/AOM/DSS + Z (60) group and ETBF colonized AOM/DSS group not given zerumbone were significantly different. There were more Bacteroides in ETBF/AOM/DSS + Z (60) group than those in ETBF colonized AOM/DSS group, suggesting that $B$. fragilis could be a normal flora activated by zerumbone. In addition, based on linear discriminant analysis of effect size (LEfSe) analysis, microbial diversity decreased significantly in the ETBF colonized AOM/DSS group. However, after given zerumbone, the taxonomic relative abundance was increased. These findings suggest that zerumbone not only influenced the microbial diversity and richness, but also could be helpful for enhancing the balance of gut microbial composition. In this work, we demonstrate that zerumbone could restore the composition of intestinal microbiota.

Keywords: Colorectal cancer, ETBF, inflammation, microbial diversity, zerumbone

Received: June 23, 2020 Accepted: September 10, 2020

First published online: September 14, 2020

*Corresponding author Phone: +82-41-530-3039 Fax: +82-41-530-3085 E-mail: omnibin@sch.ac.kr

pISSN 1017-7825 elSSN $1738-8872$

Copyright(C) 2020 by The Korean Society for Microbiology and Biotechnology

\section{Introduction}

Colorectal cancer (CRC) is the third most common cancer and the fourth leading cause of common malignant neoplasm worldwide, accounting for approximately 1.2 million new cases and 600,000 deaths per year [1,2], The common use of fecal samples to study microbial composition may reflect disease state [3]. With advance in metagenomic technology, growing evidence now suggests that dysbiosis, i.e., disorder of normal intestinal microbiota, can promote inflammatory conditions and production of carcinogenic metabolites $[4,5]$.

The gut commensal microbiota as a mutualistic ecosystem plays vital roles in maintaining host health such as digestion of dietary components, production of metabolites (fatty acids, vitamins), and inducing disease [6-8]. Many studies have analyzed compositions of intestinal microbes to identify various disorders such as inflammatory bowel diseases (IBD), colon cancer, obesity, and diabetes [9-11]. The most abundantly phylum found in the body include Firmicutes, Bacteroidetes, Actinobacteria, and Proteobacteria [12]. Among these phyla, we have used Bacteroides fragilis (B. fragilis), a species belonging to phylum Bacteroidetes, and zerumbone, a compound with anti-bacterial and anti-biofilm effect on $B$. fragilis $[13,14]$, to demonstrate that zerumbone could balance the composition of intestinal microbiota in microbial dysbiosis. Gram-negative Bacteroides species are most dominant constituents of the gut microbiota [15]. As a representative species belonging to Bacteroides, $B$. fragilis is divided into two subtypes: non-enterotoxigenic B. fragilis (NTBF) and enterotoxigenic B. fragilis (ETBF). Recently, non-toxigenic B. fragilis (NTBF) has been shown to have health benefits for the host. It is regarded as a probiotic candidate [16-18]. Wang et al. have suggested that $B$. fragilis will be the first probiotic species from the phylum Bacteroidetes [19]. 
Medical herbs and plant foods contain many biologically active phytochemicals that have various healthpromoting effects [20]. Zerumbone is a main component of the edible ginger plant Zingiber zerumbet, originating in South-East Asia, and has been cultivated for thousands of years as a spice and for medical purposes [21]. Zingiber zerumbet contains several types of phytochemical and is considered as one of the most generally used traditional dietary seasoning throughout Asia [22].

Recent studies revealed several biological properties of zerumbone that may be responsible for inhibition of ETBF-induced colon carcinogenesis. Zerumbone has been reported to have anti-inflammatory and anti-cancer activities [23-26]. Our previous study demonstrated that zerumbone has anti-biofilm and antimicrobial effects against ETBF [14]. Apparently, it is hypothesized that intake of zerumbone may result in enhanced protection from the development of microbial dysbiosis associated with ETBF. However, to the best of our knowledge, no studies have reported preventive or balancing effects of zerumbone on ETBF-mediated dysbiosis in mice.

The purpose of this study was to determine whether oral treatment of zerumbone might have the ability to alter the composition of intestinal microbiota in mice with CRC caused by ETBF. Here, we assessed whether the zerumbone-induced improvements in gut microbiota in mice colonized with ETBF using the Illumina MiSeq platform

\section{Materials and Methods \\ Bacteriology}

The wild-type ETBF strain used in our study was B. fragilis 86-5443-2-2 (bft-2). This wild-type Bacteroides strain was resistant to gentamicin and clindamycin. Colonization of bacteria was monitored by serial dilution and plating of stool on brain heart infusion agar plates containing $50 \mu \mathrm{g} \mathrm{ml}^{-1}$ of gentamicin (Corning, USA) and $6 \mu \mathrm{g} \mathrm{ml}^{-1}$ of clindamycin (Hospira, USA). Characteristic B. fragilis colonies were enumerated after anaerobic culture and shown as colony-forming units $(\mathrm{CFU}) \mathrm{gram}^{-1}$ stool. The bacterial strain was a generous gift from Cynthia Sears and Augusto Franco-Mora (Johns Hopkins University, USA).

\section{ETBF/AOM/DSS Mouse Model}

The experimental protocol was approved by the Institutional Animal Care and Use Committee of Yonsei University at Wonju, Korea (approval number: YWCL-201901-002-01). All experiments were performed in accordance with relevant guidelines under the Institutional Animal Care and Use Committee of Yonsei University at Wonju, Korea. Female BALB/c mice at 8-week-old (Rion-bio, Republic of Korea) received a single intraperitoneal injection of $10 \mathrm{mg} \mathrm{kg}^{-1}$ of AOM (Sigma-Aldrich, USA). ETBF/AOM/DSS mouse model was constructed as described previously $[26,27]$ with minor modifications. In brief, at two days after AOM injection, mice were given water containing clindamycin $\left(100 \mathrm{mg} \mathrm{l}^{-1}\right)$ and gentamicin $\left(300 \mathrm{mg} \mathrm{l}^{-1}\right)$ for 5 days to promote colonization of $B$. fragilis. Mice were inoculated with bacteria. Antibiotic-containing water was continued for additional 7 days. After 7 days of distilled water (DW), mice were administrated with the first DSS cycle (5 days DSS, 16 days of DW) for a total of 3 cycles. DSS ( $36-50 \mathrm{kDa})$ was purchased from MP Biomedicals (USA). Bacteria were grown in brain heart infusion broth (Becton, Dickinson and Company, USA) and adjusted to $1 \times 10^{9} \mathrm{CFU}$ $200 \mu \mathrm{l}^{-1}$ for mouse oral inoculations.

\section{Zerumbone Treatment and DNA Extraction}

Zerumbone was purchased from Kingherbs (China). BALB/c mice were given AOM once (10 $\left.\mathrm{mg} \mathrm{kg}^{-1}\right), \mathrm{ETBF}$ $\left(1 \times 10^{9} \mathrm{CFU} 200 \mu \mathrm{l}^{-1}\right)$, and 2 cycles of DSS (1\%). Administration of zerumbone was initiated simultaneously with DSS treatment. During 2 cycles of DSS, BALB/c mice were treated with zerumbone $\left(60 \mathrm{mg} \mathrm{kg}^{-1}\right.$, p.o.) three times a week. Mice from each group were monitored daily for water consumption. Cecum contents were stored at $-80^{\circ} \mathrm{C}$ immediately after sample collection. DNA was extracted from all samples using FastDNA SPIN Kit for feces (MPbio, USA) according to the manufacturer's instructions and stored at $-80^{\circ} \mathrm{C}$ prior to amplification.

\section{PCR Amplification and MiSeq Sequencing}

PCR was performed for the $16 \mathrm{~S}$ rRNA hypervariable region V3-V4 using primers of 341F (5'-TCGTCGGCA GCGTCAGATGTGTATAAGAGACAG-CCTACGGGNGGCWGCAG-3', underlining sequence indicating target region primer) and 805R (5'-GTCTCGTGGGCTCGG-AGATGTGTATAAGAGACAG-GACTACHVGGGT ATCTAATCC- $3^{\prime}$ ) as described previously [28]. PCR conditions were as follows: initial denaturation at $95^{\circ} \mathrm{C}$ for 3 min followed by $25 \mathrm{cycles}$ of denaturation at $95^{\circ} \mathrm{C}$ for $30 \mathrm{sec}$, primer annealing at $55^{\circ} \mathrm{C}$ for $30 \mathrm{sec}$, and extension at $72^{\circ} \mathrm{C}$ for $30 \mathrm{sec}$, with a final elongation step at $72^{\circ} \mathrm{C}$ for $5 \mathrm{~min}$. Secondary amplification for attaching Illumina NexTera barcode was then performed with i5 forward primer (5'-AATGATACGGCGACCACCGAGATCTACACXXXXXXXXTCGTCGGCAGCGTC-3'; X indicates the barcode region) and i7 reverse primer (5'CAAGCAGAA GACGGCATACGAGAT-XXXXXXXX-GTCTCGTGGGCTCGG-3'). The condition of secondary amplification was similar to the former one except that the amplification cycle was set to 8 . The PCR product was confirmed by $1 \%$ agarose gel electrophoresis and visualized under a Gel Doc system (BioRad, Hercules, CA, USA). Amplified PCR products were purified with CleanPCR (CleanNA, Waddinxveen, Netherlands). Equal concentrations of purified products were pooled together and short fragments (non-target products) were removed with CleanPCR (CleanNA). The quality and product size were assessed on a Bioanalyzer 2100 (Agilent, USA) using a DNA 7500 chip. Mixed amplicons were pooled and sequencing was carried out at Chunlab, Inc. (Republic of Korea) with Illumina MiSeq Sequencing system (Illumina, USA) according to the manufacturer's instructions. 


\section{Microbiome Taxonomic Profiling}

Processing raw reads starts with quality check and filtering the following leads, (1) Average quality less than 25 leads (AVGQUAL:25), (2) Lead length less than 100 (MINLEN:100) using the Trimmomatic program 0.32 [29]. For quality filtering, the adapter trimming work was performed separately with ChunLab's in-house code using the merged sequence, and the average quality for each lead is calculated to remove the leads that under the standard. After QC pass, paired-end sequence data were merged together using PANDAseq [30]. Primers were then trimmed with ChunLab's in-house program at a similarity cut off of 0.8 . Nonspecific amplicons that did not encode 16S rRNA were detected by using HMMER's hmmsearch program [31] with $16 \mathrm{~S}$ rRNA profiles. Sequences were denoised using DUDE-Seq [32] and non-redundant reads were extracted by UCLUST-clustering [33]. EzBioCloud database was used for taxonomic assignment using USEARCH (8.1.1861_i86linux32) [33] followed by more precise pairwise alignment [34]. UCHIME and the non-chimeric $16 \mathrm{~S}$ rRNA database from EzBioCloud were used to detect chimera on reads that contained less than $97 \%$ best hit similarity rate. Sequence data were then clustered using CD-HIT [35] and UCLUST and defined OTUs in this step.

\section{Statistical Analysis}

Comparison of mean was performed using unpaired, two-tailed Mann-Whitney $U$ test unless otherwise indicated. All statistical analyses were performed using GraphPad Prism (GraphPad Software Inc, USA) and EzBioCloud (ChunLab, Inc, Seoul, Korea). Statistical significance was set at $p$-value $<0.05$. LEfSe analysis was used to predict how zerumbone treatment impact their composition of gut microbiome in the main functional levels (KEGG categories) [36]. LEfSe exploits the Kruskal-Wallis rank-sum test to identify significantly different characteristics between assigned taxa compared to the groups, and uses Linear discriminant analysis (LDA) to estimate the size effect, based on a LDA socre $>2.0$ and $p$-value $<0.05$. The LEfSe were analyzed by the EzBioCloud database (ChunLab, Inc.) [37]. Alpha diversity analysis expressed with ACE (http://www.mothur.org/wiki/Ace), Chao1 (http://www.mothur.org/wiki/Chao), Jackknife (http://www.mothur.org/wiki/Jack), OTUs, Shannon (http://www.mothur.org/wiki/Shannon) and Simpson (http://www.mothur.org/wiki/Simpson) were conducted using CLcommunity software (ChunLab, Inc.).

\section{Results}

\section{Effects of Zerumbone on the Composition of Gut Microbiome at Phylum Levels}

Microbial dysbiosis caused by inflammation and tumorigenesis directly contributes towards polyp formation in the AOM/DSS model [38]. It was hypothesized that zerumbone treatment led to a decrease in ETBF-mediated dysbiosis by altering microbial composition. Therefore, in order to test the hypothesis, we analyzed bacterial communities in all stool specimens to assess differences in the composition of gut microbiota between sham, ETBF colonized group, and zerumbone treated groups. 16S rRNA gene clone libraries were established and sequenced.

Taxonomic composition data at the level of phylum showed that three phyla were dominant, including Firmicutes (84.11\%), Verrucomicrobia (10.16\%), Proteobacteria (5.69\%) in sham group (Fig. 1A). Of these major phyla in fecal microbiota of the sham group, Firmicutes (84.11\%) was the most predominant phylum. Proportions of Verrumicrobia in ETBF colonized AOM/DSS, ETBF/AOM/DSS + Z (60), and zerumbone $\left(60 \mathrm{mg} \mathrm{kg}^{-1}\right)$ groups were increased $(39.51 \%, 42.81 \%, 59.23 \%$, respectively) (Figs. 1B-1D). In addition, the level of Bacteroides was higher in the ETBF/AOM/DSS + Z (60) group than that in ETBF colonized AOM/DSS group $(23.48 \%$ and $1.18 \%$, respectively, $p$-value: 0.00149$)$. Results indicated a microbial diversity was influenced and more balanced in $\mathrm{ETBF} / \mathrm{AOM} / \mathrm{DSS}+\mathrm{Z}$ (60) compared with ETBF-colonized AOM/DSS group (Fig. 1).

\section{Gut Microbiota Abundance at Family Level}

Average taxonomic compositions of MTP sets were also determined at family level (Fig. 2). We analyzed and focused on four families (Lachnospiraceae, Enterobactericeae, Akkermansiaceae, Bacteroidaceae) seen as the most predominant families in overall group. Compared to other groups, families that were the most different from sham group were Lacchnospiraceae and Akkermansiaceae. While Lacchnospiraceae decreased greatly, Akkermansiaceae showed an obvious increase (Figs. 2A and 2C). Enterobacteriaceae was significantly decreased in ETBF colonized AOM/DSS group. It was found to have high relative abundance as much as in the sham group and in ETBFcolonized AOM/DSS mice given zerumbone (Fig. 2B). Bacteroidaceae was significantly more abundant in the gut microbiota of ETBF/AOM/DSS + Z (60) group than that of ETBF colonized AOM/DSS group (Fig. 2D). Interestingly, results showed that phylum Bacteroidetes was not detected in sham or zerumbone $\left(60 \mathrm{mg} \mathrm{kg}^{-1}\right)$ group, although it was detected at low portion in the ETBF colonized AOM/DSS group and increased in the ETBF/ $\mathrm{AOM} / \mathrm{DSS}+\mathrm{Z}$ (60) group at phylum level. Similarly, family Bacteroidaceae was increased in the ETBF/AOM/DSS $+\mathrm{Z}(60)$ group.

\section{Microbial Compositions for Each Group at Genus and Species Levels}

To further determine microbiome populations at genus level, relatively balanced microbial composition was observed in the ETBF/AOM/DSS $+\mathrm{Z}$ (60) group during the experiment (Fig. 3A). Akkermansia rapidly increased in the zerumbone $\left(60 \mathrm{mg} \mathrm{kg}^{-1}\right)$ group (59.23\%). We observed a relative abundance of Clostridium_g24 in sham group (54.64\%). It was significantly reduced in the ETBF colonized AOM/DSS group to $1.57 \%$. It was then enriched after given zerumbone (9.74\%). Like the phylum level, the proportion of Bacteroides was higher in the $\mathrm{ETBF} / \mathrm{AOM} / \mathrm{DSS}+\mathrm{Z}$ (60) group (23.49\%) than that in the ETBF colonized AOM/DSS group (1.16\%). Compared 
A

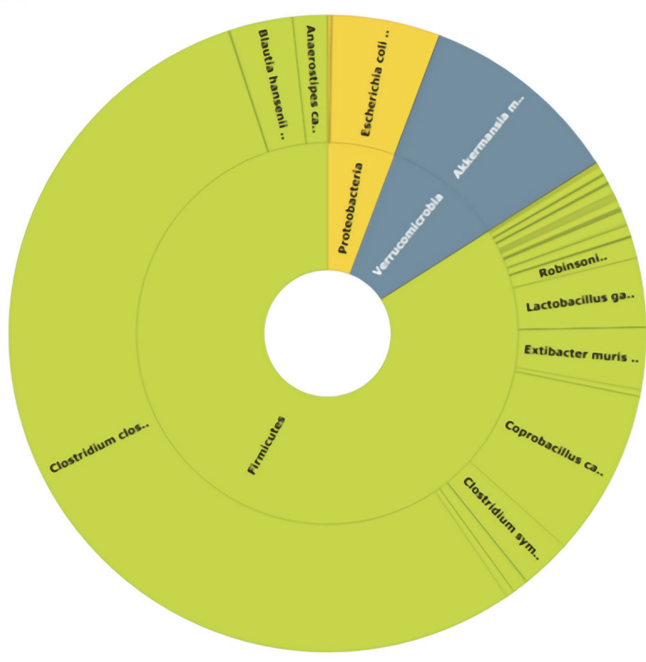

Firmicutes (84.11\%)

Proteobacteria (5.69\%)

Verrucomicrobia (10.16\%)

ETC(under $1 \%$ in average) $(0.04 \%)$

ETBF/AOM/DSS +Z(60)

C

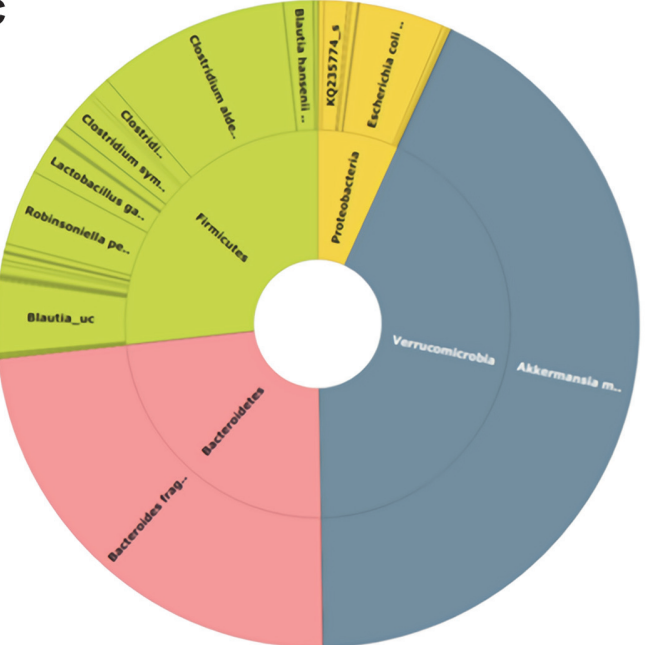

Firmicutes (26.85\%)

Proteobacteria (6.85\%)

Verrucomicrobia (42.81\%)

Bacteroidetes (23.48\%)

ETC(under $1 \%$ in average) $(0.01 \%)$
B

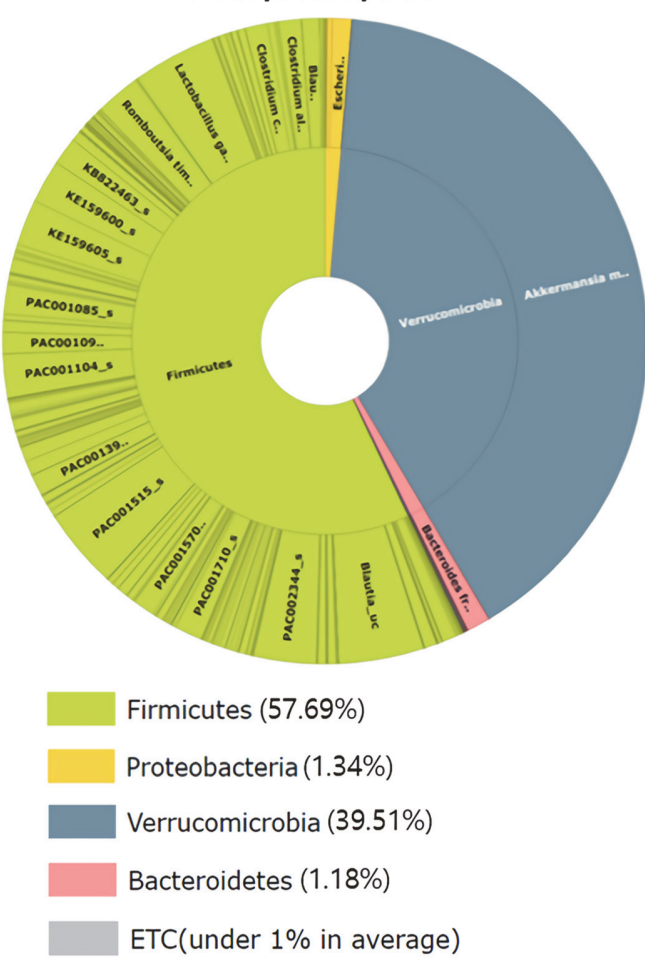

$Z(60)$

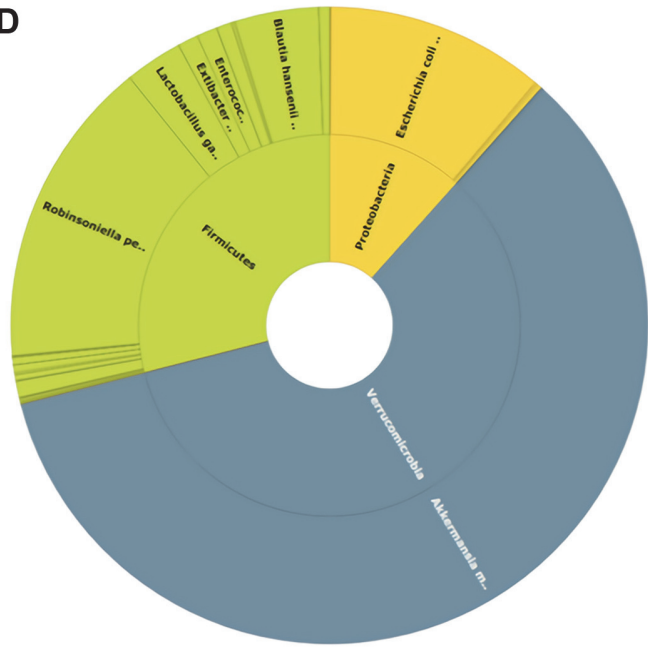

Firmicutes (29.10\%)

Proteobacteria (11.65\%)

Verrucomicrobia (59.23\%)

ETC(under $1 \%$ in average) $(0.02 \%)$

Fig. 1. Bacterial community composition of cecum contents in each group. The relative abundances of $16 \mathrm{~S}$ rRNA gene sequences (V3-V4 region), classified to the phylum (inner pie) and species (outer pie) level, are shown. Firmicutes, the phylum to which Clostridium clostridioforme group belongs was the major phylum in (A) sham control and (B) ETBFcolonized AOM/DSS group. Verrucomicrobia the phylum to which Akkermansia muciniphila belongs was the major phylum in (C) ETBF-colonized AOM/DSS group administered with $60 \mathrm{mg} \mathrm{kg}^{-1}$ zerumbone and (D) control group administered with $60 \mathrm{mg} \mathrm{kg}^{-1}$ zerumbone. Abbreviations of group names are as follows: Sham, sham control; ETBF/AOM/DSS, ETBF colonized $\mathrm{AOM} / \mathrm{DSS}$; ETBF/AOM/DSS + Z(60), ETBF colonized AOM/DSS given zerumbone $\left(60 \mathrm{mg} \mathrm{kg}^{-1}\right) ; \mathrm{Z}(60)$, zerumbone $\left(60 \mathrm{mg} \mathrm{kg}^{-1}\right)$. 


\section{A Lachnospiraceae}

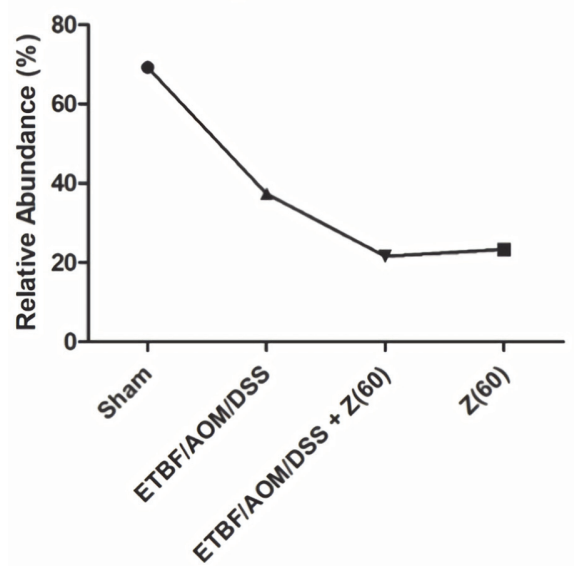

C Akkermansiaceae

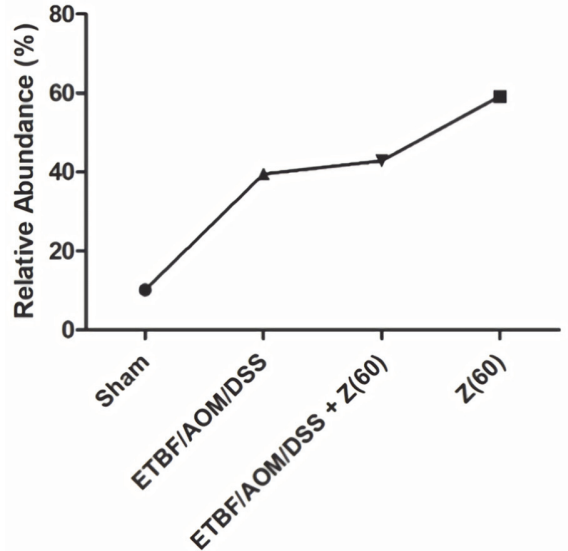

B Enterobacteriaceae

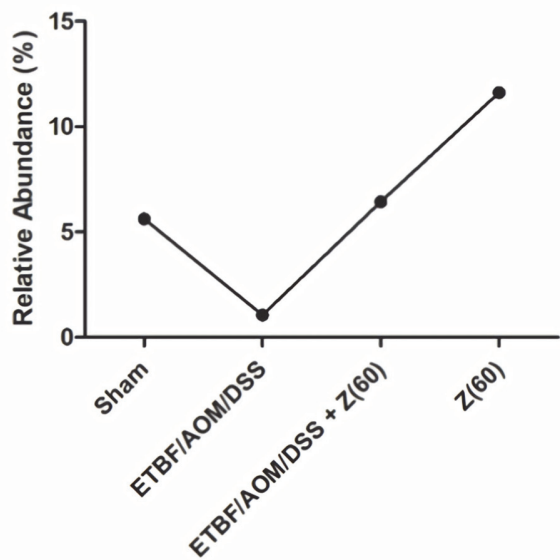

D Bacteroidaceae

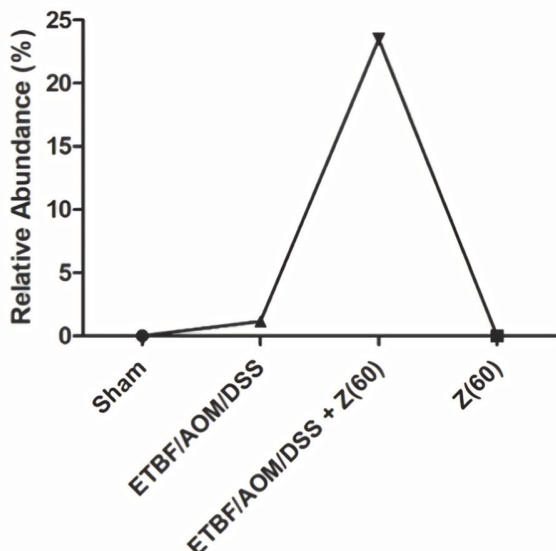

Fig. 2. Gut microbiota at family level (Lachnospiraceae, Akkermansiaceae, Enterobactericeae, Bacteroidaceae). Significant families of each group based on $16 \mathrm{~S}$ rRNA sequencing. Abbreviations of group names are as follows: Sham, sham control; ETBF/AOM/DSS, ETBF colonized AOM/DSS; ETBF/AOM/DSS +Z(60), ETBF colonized AOM/DSS given zerumbone $\left(60 \mathrm{mg} \mathrm{kg}^{-1}\right) ; \mathrm{Z}(60)$, zerumbone $\left(60 \mathrm{mg} \mathrm{kg}^{-1}\right)$.

to other groups, various strains were seen in ETBF colonized AOM/DSS group. In addition, the ETC (under 1\% in average) was significantly increased (13.92\%).

In order to investigate changes in gut microbiota in detail, averaged taxonomic compositions of each group were analyzed at species levels based on the EzTaxon-e database [33]. In ETBF-colonized AOM/DSS group, the most abundant species was Akkermansia muciniphila (40.11\%), followed by Blautia_uc (4.28\%), and Lactobacillus gasseri (4.19\%). On the other hand, the most abundant species was Akkermansia muciniphila (42.88\%), followed by Bacteroides fragilis (23.52\%), and Clostridium aldenense (9.64\%) in ETBF/AOM/DSS + Z (60) (Fig. 3B). It was apparent that zerumbone-induced interventions affecting the composition of the intestinal microbiota may be a strategy to prevent the microbial dysbiosis prompted by ETBF.

\section{Taxonomic Biomarker Discovery Based on LEfSe Analysis of Gut Microbiota among Four Groups}

We also performed LEfSe analysis at various level to assess which microbiota were driving divergence between different groups using parameters described above (Fig. 4). LEfSe analysis revealed that not only phylum Verrucomicrobia and its derivative class Verrucimicrobiae, order Verrucomicrobiales, but also family Akkermansiaceae were significantly higher in the zerumbone $\left(60 \mathrm{mg} \mathrm{kg}^{-1}\right)$ group and ETBF/AOM/DSS + Z (60) group, but significantly lower in the intestinal microbiota from sham group (LDA score: 4.216, $p$-value: 0.00423). Additionally, LEfSe analysis revealed that phylum Firmicutes (LDA score: 4.051, p-value: 0.00168), class Clostridia and order Clostridiales (LDA score: 4.01, p-value: 0.00182 ) were significantly higher in sham and ETBF colonized AOM/DSS groups. Especially, Clostridium g24 genus was significantly higher in the sham group (LDA score: 3.890 , $p$-value: 0.00089 ). 

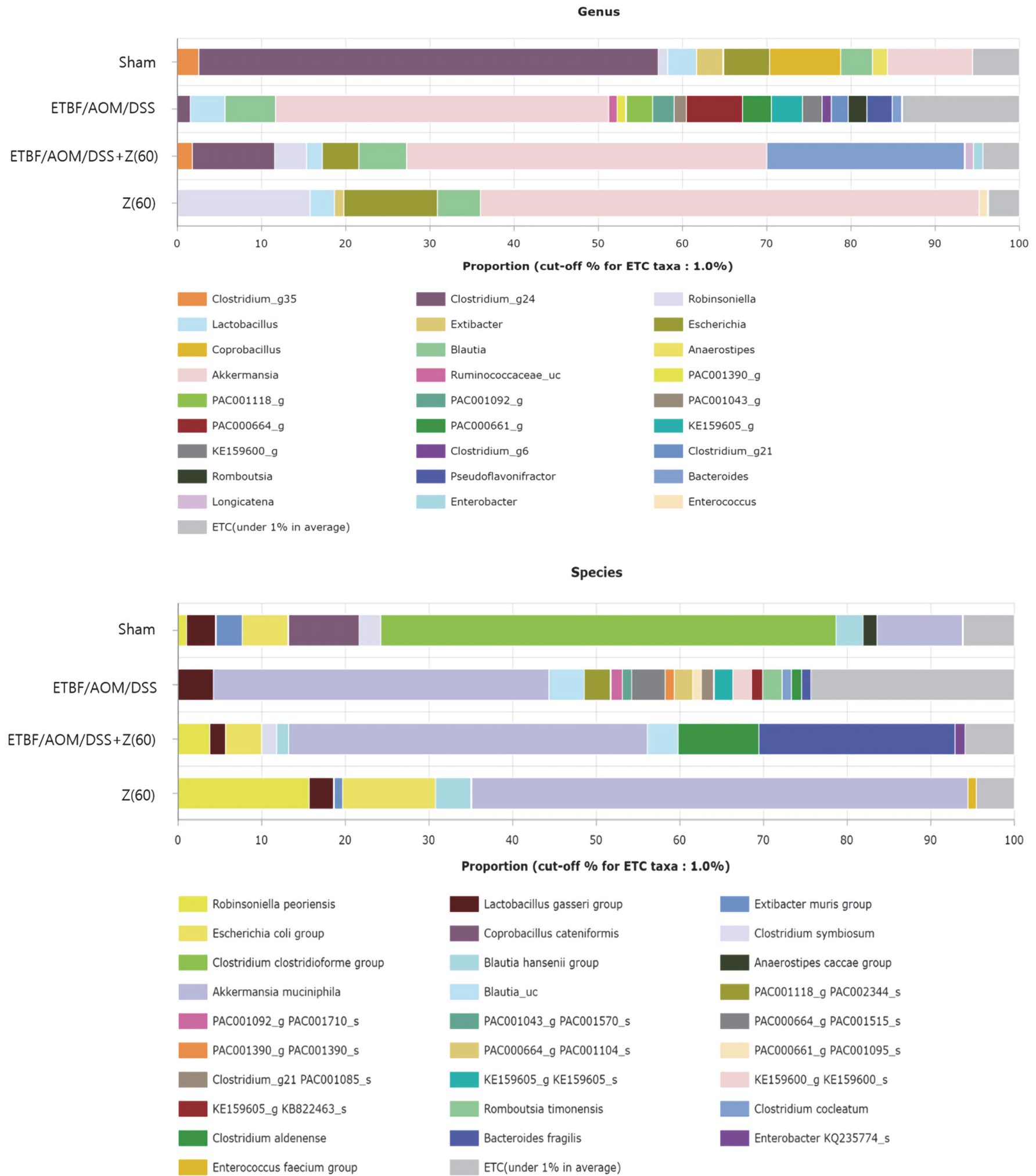

Fig. 3. The proportion of gut microbiome at genus and species levels in each group. (A) Proportion of gut microbiome at genus level. (B) Averaged taxonomic compositions at species levels. Nomenclature is based on the EzTaxon-e database. Abbreviations of group names are as follows: Sham, sham control; ETBF/AOM/DSS, ETBF colonized AOM/DSS;ETBF/AOM/DSS + Z(60), ETBF colonized AOM/DSS given zerumbone $\left(60 \mathrm{mg} \mathrm{kg}^{-1}\right) ; \mathrm{Z}(60)$, zerumbone $\left(60 \mathrm{mg} \mathrm{kg}{ }^{-1}\right)$. 
A Verrucomicrobia Verrucomicrobiae Verrucomicrobiales Akkermansiaceae Akkermansia

Akkermansia muciniphila Verrucomicrobia Verrucomicrobiae Verrucomicrobiales Akkermansiaceae Akkermansia Akkermansia muciniphila Verrucomicrobia Verrucomicrobiae Verrucomicrobiales Akkermansiaceae Akkermansia Akkermansia muciniphila Firmicutes Clostridia Clostridiales Lachnospiraceae Firmicutes Clostridia Clostridiales achnospiraceae Clostridium g24

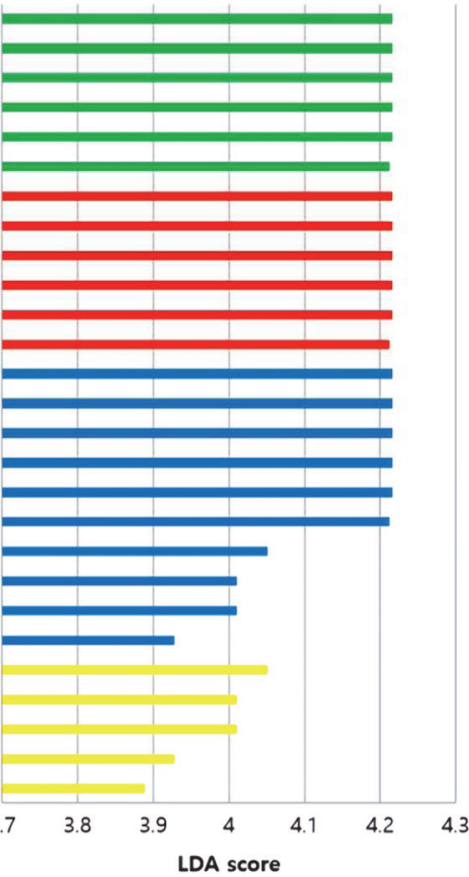

$Z(60)$

$E T B F / A O M / D S S+Z(60)$

ETBF/AOM/DSS

Sham
B

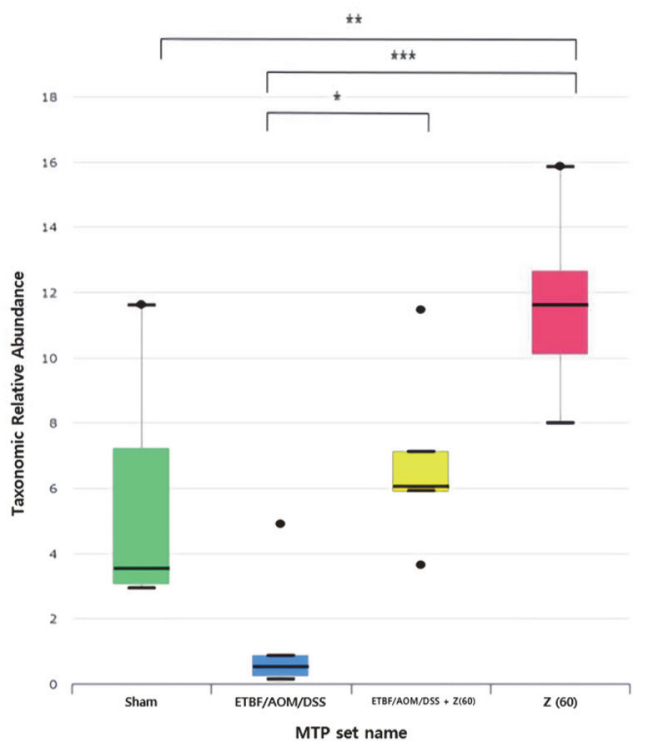

Fig. 4. LEfSe analysis of different abundance levels. (A) Histogram of LDA scores for different abundance levels. LDA scores represent the effect size of each abundance level. Levels enriched in each group with an LDA score $>2$ are considered. (B) Taxonomic relative abundant box plot of Proteobacteria. LDA score was calculated using LEfSe, a metagenome analysis approach. Abbreviations of group names are as follows: Sham, sham control; ETBF/AOM/DSS, ETBF colonized $\mathrm{AOM} / \mathrm{DSS} ; \mathrm{ETBF} / \mathrm{AOM} / \mathrm{DSS}+\mathrm{Z}(60)$, ETBF colonized AOM/DSS given zerumbone $\left(60 \mathrm{mg} \mathrm{kg}^{-1}\right) ; \mathrm{Z}(60)$, zerumbone (60 mg kg $\left.{ }^{-1}\right)$.

\section{Alpha Diversity of Gut Microbiota in Each Group}

We compared alpha diversity between groups. Alpha diversity is known to reflect the richness and evenness by measuring the number of OTUs found in MTP, Shannon diversity index etc. based on $16 \mathrm{~S}$ rRNA gene sequencing data. Alpha diversity evaluation among the four groups using six different metrics are shown in Fig. 5. We used values given by one or more diversity index such as species richness (ACE, Chao 1, Jackknife, number of OTUs found in MTP) and diversity index (Shannon index or the Simpson index). Overall, alpha diversity scores tended to be higher (except the Simpson index) for the ETBF colonized AOM/DSS group but lower for the sham group, $\mathrm{ETBF} / \mathrm{AOM} / \mathrm{DSS}+\mathrm{Z}(60)$ group, and zerumbone $\left(60 \mathrm{mg} \mathrm{kg}^{-1}\right)$ group. OTU measurements and Shannon Index of ETBF colonized AOM/DSS group were significantly increased, demonstrating that there was richness and various strains. Whereas in zerumbone group and sham group, the diversity decreased significantly. The ETBF/AOM/ DSS + Z (60) group showed a tendency to be similar to sham group. These results indicate that zerumbone can restore changes induced by AOM/DSS treatment to decrease/increase the abundance of these microbial species so that they can resemble the sham group.

\section{Assessment of Group Similarities in Species Composition by Beta Diversity}

Beta diversity was assessed using principal coordinates analysis (PCoA) scores and UPGMA dendrogram (Fig. 6). PCoA based on the relative abundance of between groups of samples revealed that a significant separation in bacterial community composition using the first three principal coordinates scores of PC1, PC2, and PC3. The gut microbiota of each group was significantly different from that of other groups based on both PCoA scores plot and UPGMA dendrogram. In addition, we could identify each sample was clustered by subject. PCoA and dendrogram showed almost the same results. From the result that zerumbone $\left(60 \mathrm{mg} \mathrm{kg}^{-1}\right)$ group and ETBF/ $\mathrm{AOM} / \mathrm{DSS}+\mathrm{Z}(60)$ group were relatively close in PCoA and that they were almost on one plane, it could be concluded that compositions of strains between groups were similar (Fig. 6A). The only zerumbone-treated group indicated distinct position compared to sham control in PCoA score, suggesting that zerumbone treatment changes microbial community with or without inflammation or tumorigenesis. In dendrogram, sham group seemed to have a different cluster from that of other groups based on Fast Unifrac distance (Fig. 6B). Beta diversity results based on $\mathrm{PCoA}$ and dendrogram demonstrated similarities in bacterial community structure among $\mathrm{ETBF} / \mathrm{AOM} / \mathrm{DSS}+\mathrm{Z}(60)$ group and zerumbone group.

\section{Assessment of Firmicutes to Bacteroidetes Ratio (F/B Ratio)}

We analyzed the F/B ratio with given result between ETBF/AOM/DSS + Z (60) group and ETBF colonized AOM/DSS group (Fig. 7). The F/B ratio of the ETBF colonized AOM/DSS group was much higher than that of the ETBF colonized AOM/DSS given zerumbone. This means that there is a bowel disease in the ETBF colonized AOM/DSS group. 
ACE
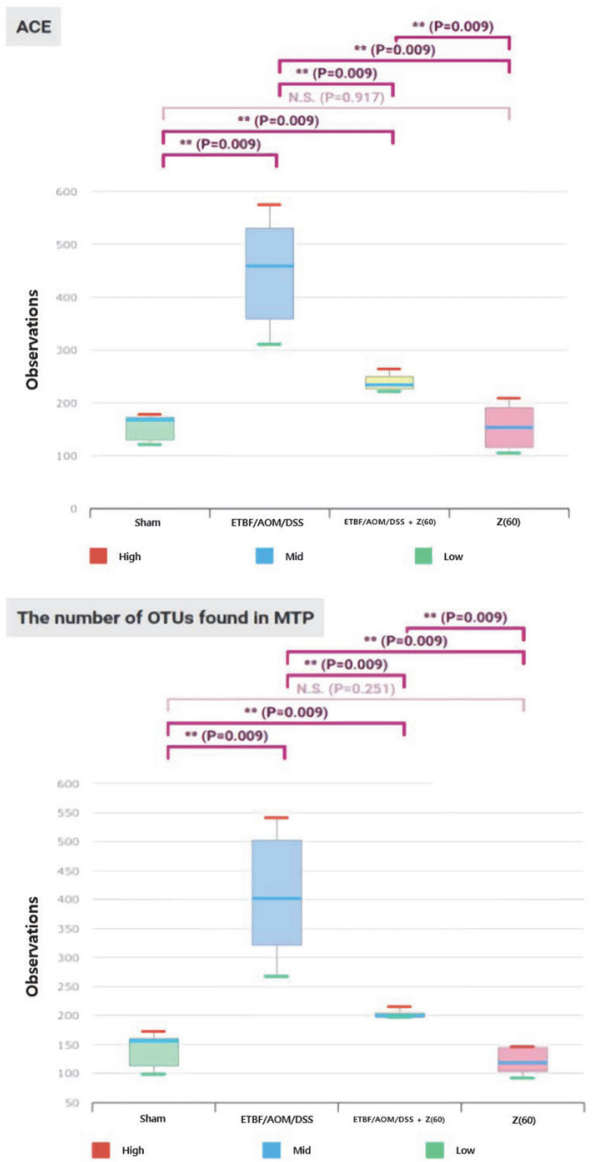
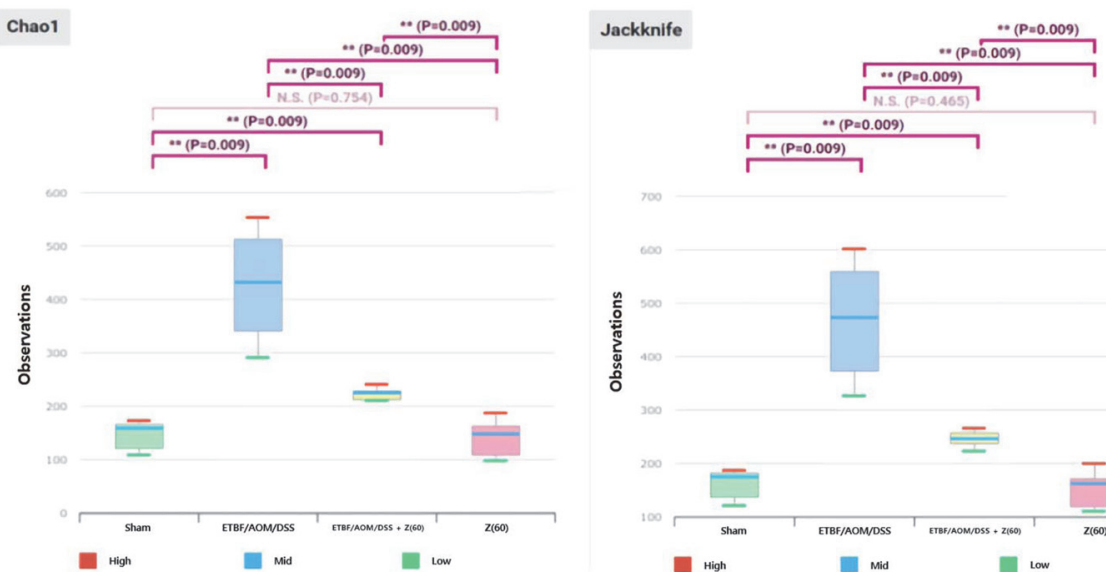

Shannon
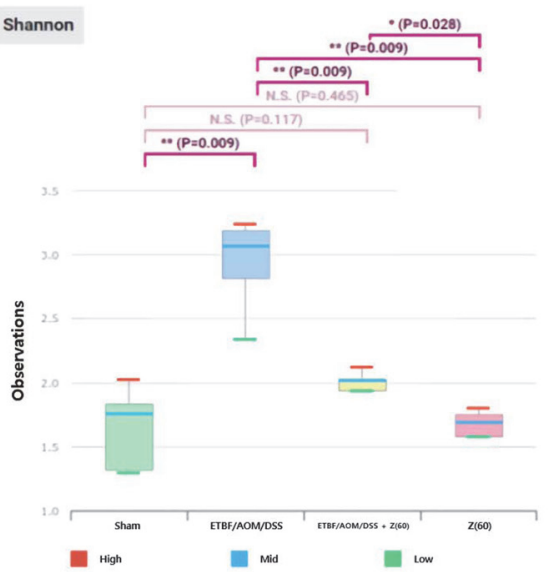
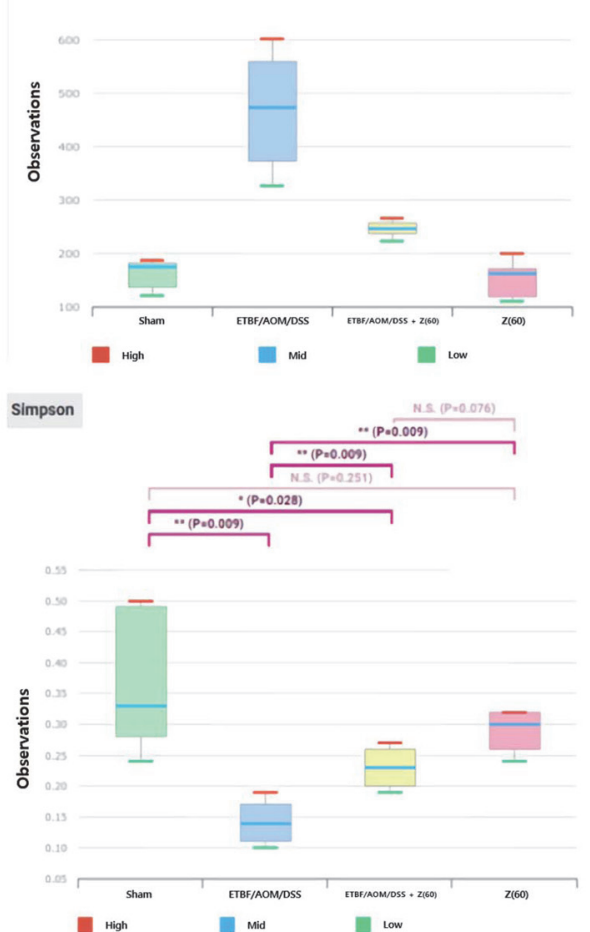

Fig. 5. Alpha-diversity calculated using phylotype relative abundance measurrements in each group. Box plot shows similar aspest among each group. ETBF/AOM/DSS group tended to be higher than other groups. We measured ACE, Chaol, Jackknife, the number of OTUs found in MTP, Shannon and Simpson index based on 16S rRNA gene sequencing data. Abbreviations of group names are as follows: Sham, sham control; ETBF/AOM/DSS, ETBF colonized AOM/DSS; ETBF Z(60), ETBF colonized AOM/DSS given zerumbone $\left(60 \mathrm{mg} \mathrm{kg}^{-1}\right)$; Z, zerumbone $\left(60 \mathrm{mg} \mathrm{kg}^{-1}\right)$.

A

Principal coordinates analysis [3D] [Jensen-Shannon, Species, Include Unclassified OTUs (Reads)]

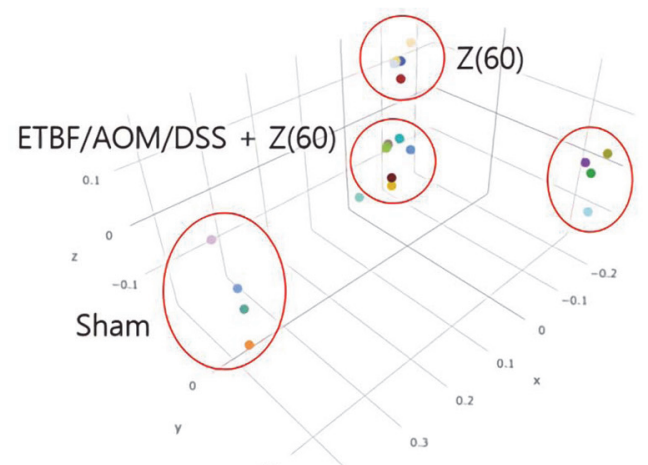

ETBF/AOM/DSS

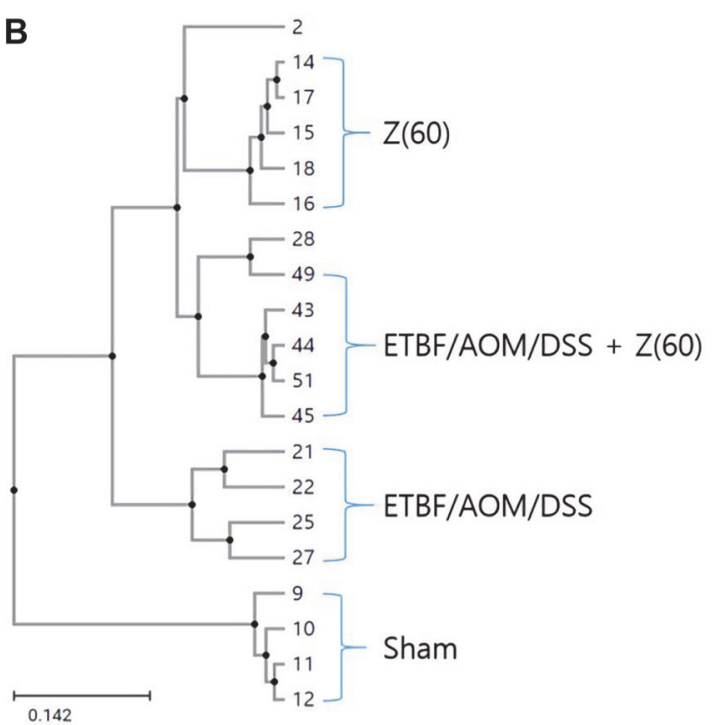

Fig. 6. Assessment of group similarities in species composition by beta diversity. (A) 3D principal coordinates analysis (PCoA) of Sham, ETBF, ETBF $+Z(60)$, Z (60). Each point based on principal component score 1, 2, and 3 of all samples in a group. (B) UPGMA dendrogram. Each sample was clustered by subject. Abbreviations of group names are as follows: Sham, sham control; ETBF/AOM/DSS, ETBF colonized AOM/DSS; ETBF/AOM/DSS + Z(60), ETBF colonized AOM/ DSS given zerumbone $\left(60 \mathrm{mg} \mathrm{kg}^{-1}\right) ; \mathrm{Z}(60)$, zerumbone $\left(60 \mathrm{mg} \mathrm{kg}^{-1}\right)$. 

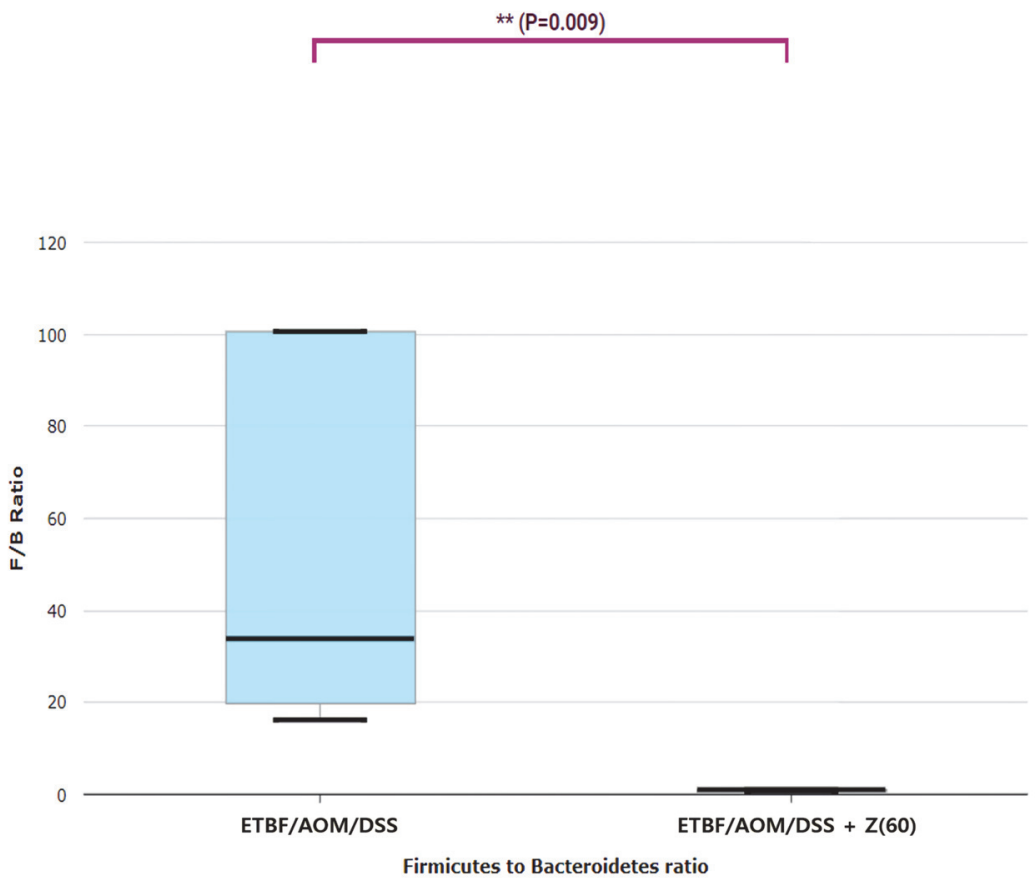

Fig. 7. Firmicutes to Bacteroidetes ratio (F/B ratio). F/B ratio of ETBF colonized AOM/DSS and ETBF colonized AOM/DSS given zerumbone $\left(60 \mathrm{mg} \mathrm{kg}^{-1}\right)$. The F/B ratio of ETBF colonized AOM/DSS was significantly higher than that of $\mathrm{ETBF} / \mathrm{AOM} / \mathrm{DSS}+\mathrm{Z}(60)$ group. Abbreviations of group names are as follows: ETBF/AOM/DSS, ETBF colonized AOM/DSS; $E T B / A O M / D S S+Z(60)$, ETBF colonized AOM/DSS given Zerumbone $\left(60 \mathrm{mg} \mathrm{kg}^{-1}\right)$.

\section{Discussion}

In this study, to know how fecal microbiota might change after given zerumbone, we first analyzed compositions of gut microbiota and determined whether zerumbone could restore gut microbiota of ETBFcolonized AOM/DSS group. Our results revealed significant difference of intestinal microbiota composition between ETBF/AOM/DSS + Z (60) group and ETBF colonized AOM/DSS group. The relative abundance of dominant phyla Firmicutes, Proteobacteria, Verrucomicrobia, Bacteroides (Fig. 1), and dominant families Lachnospiraceae, Enterobactericeae, Akkermansiaceae, Bacteroidaceae were found (Fig. 2).

Firmicutes is one of the dominant phyla within healthy adult microbiota. A quantitative reduction of this phylum has been shown in inflammatory gut disease [39]. Additionally, as observed in humans, python gut, and a wide variety of other mammals, most sequences were classified as Firmicutes $(61.8 \%)[40,41]$. Our results showed that phylum Firmicutes was the most abundant in the sham group while it was the least abundant in the two zerumbone treated groups (ETBF/AOM/DSS + Z (60) and zerumbone). The ETBF colonized AOM/DSS group had higher abundance of Firmicutes than the groups given zerumbone. A previous study has shown that healthy intestinal microbiota is predominantly constituted by phyla Firmicutes and Bacteroidetes [42]. Referred to contents of the previous study, microbiome detection in sham group is predicted to be normal flora. After processing ETBF/AOM/DSS, the formation of CRC resulted in a significantly decrease in Firmicutes and zerumbone treatment showed reduction in the number of species involved in inflammatory reaction.

Microbiome taxonomic profiling (MTP) results showed that microbial dysbiosis was improved in ETBF/AOM/ DSS $+\mathrm{Z}(60)$. The second highest species of gut microbes in the cecum of ETBF/AOM/DSS $+\mathrm{Z}(60)$ was Bacteroides fragilis (Fig. 3) compared to other groups. A recent study showed that non-toxigenic Bacteroides fragilis (NTBF), a microbe of the normal microbiota of the human colonic commensal [43], diminished ETBFinduced colitis and colitis-promoted tumorigenesis in Min mice [44]. Collectively, these results suggest that increased NTBF by oral treatment of zerumbone may tamper ETBF-promoted tumorigenesis in AOM/DSS mice.

It is definite that NTBF and ETBF cannot be classified by only 300-bp 16S rRNA gene fragments. To distinguish the NTBF and ETBF, we took phenotypic test in previous study $[45,46]$. We used B. fragilis 86-5443-2-2 (bft-2) as ETBF strain in the current study, which is resistant to gentamicin and clindamycin. Mouse stool was monitored by serial dilution and inoculating of stool on brain heart infusion agar (BHIA) plates including $50 \mu \mathrm{g} / \mathrm{ml}$ of gentamicin and $6 \mu \mathrm{g} / \mathrm{ml}$ of clindamycin. Plates were incubated overnight at $37^{\circ} \mathrm{C}$ under anaerobic conditions. Other fecal bacteria including NTBF were prevented by addition of gentamicin and clindamycin. Characteristic ETBF colonies were enumerated after anaerobic culture and shown as colony-forming units (CFU)/g stool.

Proteobacteria known to be less abundant in CRC patients are generally regarded as intestinal normal flora with potential-pathogenic features [47]. Our results showed a similar pattern to the previous research. The proportion of Proteobacteria in the ETBF colonized AOM/DSS group was the lowest (1.34\%). It was the most abundant in the 
zerumbone-treated group and the other two groups (sham and ETBF/AOM/DSS + Z (60)) were almost similar proportion. In addition, referring to LEfSe analysis, the microbial diversity of Proteobacteria dropped significantly in the ETBF/AOM/DSS group. However, after given zerumbone, the taxonomic relative abundance was increased to be as much as sham (Fig. 4). These results demonstrate that zerumbone could restore compositions of intestinal microbiota in mice with CRC caused by ETBF.

In mice and a wide variety of other mammals, most gut microbiome sequences were detected as either Firmicutes (61.8\%) or Bacteroidetes (20.6\%) [41]. According to a recent study, normal human gut microbiota comprises of two major phyla, namely Bacteroidetes and Firmicutes [42]. In addition, Chan et al. have found that non-toxigenic $B$. fragilis (NTBF) can mitigate colitis and tumorigenesis initiated by ETBF as long as NTBF introduction precedes ETBF strain exposure and remains the dominant B. fragilis strain [44]. In our study, Bacteroidetes was more abundant in the ETBF/AOM/DSS + Z (60) than ETBF colonized AOM/DSS. Based on these results, we could conclude that increase in Bacteroidetes of ETBF/AOM/DSS + Z (60) group reflects an effect of NTBF by oral treatment.

One study has found that Lactobacillus salivarius strain UCC118 belonging to phylum Firmicutes can produce a two-component bacteriocin against bacteria of Bacteroidetes [48]. In addition, Guinane et al. revealed that bactofencin $A$ has a potentially positive influence on both intestinal communities and anaerobic microbiome such as Bacteroides [49]. The ETBF/AOM/DSS + Z (60) group showed many normal strains of Bacteroidetes. It might be attributed to decreased production of bacteriocin in Firmicutes.

The Firmicutes to Bacteroidetes ratio (F/B ratio) has been used as an indicator of disease and extensively examined for human and mouse gut microbiota [50]. Also, cooperation between Firmicutes and Bacteroidetes in the body mass index has been reported [51]. An increase in Firmicutes characterizes obesity [52]. An increase in Bacteroidetes has been reported to occur in those aged 30 years and older [53]. Many studies have shown that the $\mathrm{F} / \mathrm{B}$ ratio is correlated with diseases. In our study, the F/B ratio of the ETBF/AOM/DSS + Z (60) group was almost 1 (Fig. 7). Therefore, this potentially means that zerumbone can regulate Firmicutes/Bacteroides composition and consequently improve intestinal microbial dysbiosis by balancing.

In previous studies demonstrated that zerumbone prevented both biofilm formation and biofilms pre-formed by ETBF [54], and reported that zerumbone has anti-inflammatory effect of ETBF infection without affecting ETBF colonization $[45,46]$. However, further replication studies, to clarify the prevention and to examine the balancing effects of zerumbone in the pharmaceutical field, should follow in a near future.

In conclusion, our study showed increased diversity and richness in ETBF/AOM/DSS + Z (60) compared with ETBF colonized AOM/DSS group through a number of analytical parameters, including alpha or beta diversity. Additionally, our results significantly indicated that zerumbone can ease intestinal microbial imbalance against ETBF and increase intestinal microbial diversity. Therefore, zerumbone is proposed as a promising natural preventive agent along with conventional antimicrobial agents against ETBF-associated colorectal cancer with the potential to maintain human health via dietary supplementation.

\section{Acknowledgments}

This study was supported by the Soonchunhyang University research fund and the National Research Foundation of Korea (NRF) grant funded by the Korea government (MSIT) [NRF-2020R1F1A1071977].

\section{Conflict of Interest}

The authors have no financial conflicts of interest to declare.

\section{References}

1. Siegel RL, Miller KD, Jemal A. 2018. Cancer statistics, 2018. CA Cancer J. Clin. 68: 7-30.

2. Brenner H, Kloor M, Pox CP. 2014. Colorectal cancer. Lancet 383: 1490-1502.

3. Thomas AM, Jesus EC, Lopes A, Aguiar S, Jr., Begnami MD, Rocha RM, et al. 2016. Tissue-associated bacterial alterations in rectal carcinoma patients revealed by $16 \mathrm{~S}$ rRNA community profiling. Front. Cell. Infect. Microbiol. 6: 179.

4. Joossens M, Huys G, Cnockaert M, De Preter V, Verbeke K, Rutgeerts P, et al. 2011. Dysbiosis of the faecal microbiota in patients with Crohn's disease and their unaffected relatives. Gut. 60: 631-637.

5. Marchesi JR, Dutilh BE, Hall N, Peters WH, Roelofs R, Boleij A, et al. 2011. Towards the human colorectal cancer microbiome. PLoS One 6: e20447.

6. Brennan CA, Garrett WS. 2016. Gut microbiota, inflammation, and colorectal cancer. Annu. Rev. Microbiol. 70: $395-411$.

7. Belkaid Y, Hand TW. 2014. Role of the microbiota in immunity and inflammation. Cell 157: 121-141.

8. Arthur JC, Perez-Chanona E, Muhlbauer M, Tomkovich S, Uronis JM, Fan TJ, et al. 2012. Intestinal inflammation targets cancerinducing activity of the microbiota. Science 338: 120-123.

9. Wang CZ, Huang WH, Zhang CF, Wan JY, Wang Y, Yu C, et al. 2018. Role of intestinal microbiome in American ginseng-mediated colon cancer prevention in high fat diet-fed AOM/DSS mice [corrected]. Clin. Transl. Oncol. 20: 302-312.

10. Janem WF, Scannapieco FA, Sabharwal A, Tsompana M, Berman HA, Haase EM, et al. 2017. Salivary inflammatory markers and microbiome in normoglycemic lean and obese children compared to obese children with type 2 diabetes. PLoS One 12: e0172647.

11. Ley RE, Backhed F, Turnbaugh P, Lozupone CA, Knight RD, Gordon JI. 2005. Obesity alters gut microbial ecology. Proc. Natl. Acad. Sci. USA 102: 11070-11075.

12. Sun J, Kato I. 2016. Gut microbiota, inflammation and colorectal cancer. Genes Dis. 3: 130-143.

13. Santosh Kumar SC, Srinivas P, Negi PS, Bettadaiah BK. 2013. Antibacterial and antimutagenic activities of novel zerumbone analogues. Food Chem. 141: 1097-1103.

14. Kim HR, Rhee KJ, Eom YB. 2019. Anti-biofilm and antimicrobial effects of zerumbone against Bacteroides fragilis. Anaerobe 57: 99-106.

15. Kononen E, Asikainen S, Jousimies-Somer H. 1992. The early colonization of gram-negative anaerobic bacteria in edentulous infants. Oral. Microbiol. Immunol. 7: 28-31.

16. Troy EB, Kasper DL. 2010. Beneficial effects of Bacteroides fragilis polysaccharides on the immune system. Front. Biosci. 15: 25-34. 
17. Hsiao EY, McBride SW, Hsien S, Sharon G, Hyde ER, McCue T, et al. 2013. Microbiota modulate behavioral and physiological abnormalities associated with neurodevelopmental disorders. Cell 155: 1451-1463.

18. Deng H, Li Z, Tan Y, Guo Z, Liu Y, Wang Y, et al. 2016. A novel strain of Bacteroides fragilis enhances phagocytosis and polarises M1 macrophages. Sci. Rep. 6: 29401.

19. Wang Y, Deng H, Li Z, Tan Y, Han Y, Wang X, et al. 2017. Safety evaluation of a novel strain of Bacteroides fragilis. Front. Microbiol. 8: 435

20. Kwon KH, Barve A, Yu S, Huang MT, Kong AN. 2007. Cancer chemoprevention by phytochemicals: potential molecular targets, biomarkers and animal models. Acta. Pharmacol. Sin. 28: 1409-1421.

21. Yob NJ, Jofrry SM, Affandi MM, Teh LK, Salleh MZ, Zakaria ZA. 2011. Zingiber zerumbet (L.) Smith: A review of its ethnomedicinal, chemical, and pharmacological uses. Evid. Based. Complement. Alternat. Med. 2011: 543216.

22. Rahman HS, Rasedee A, Yeap SK, Othman HH, Chartrand MS, Namvar F, et al. 2014. Biomedical properties of a natural dietary plant metabolite, zerumbone, in cancer therapy and chemoprevention trials. Biomed. Res. Int. 2014: 920742.

23. Moreira da Silva T, Pinheiro CD, Puccinelli Orlandi P, Pinheiro CC, Soares Pontes G. 2018. Zerumbone from Zingiber zerumbet (L.) smith: a potential prophylactic and therapeutic agent against the cariogenic bacterium Streptococcus mutans. BMC Complement Altern. Med. 18: 301.

24. Yodkeeree S, Sung B, Limtrakul P, Aggarwal BB. 2009. Zerumbone enhances TRAIL-induced apoptosis through the induction of death receptors in human colon cancer cells: Evidence for an essential role of reactive oxygen species. Cancer Res. 69: 6581-6589.

25. Sulaiman MR, Perimal EK, Akhtar MN, Mohamad AS, Khalid MH, Tasrip NA, et al. 2010. Anti-inflammatory effect of zerumbone on acute and chronic inflammation models in mice. Fitoterapia 81: 855-858.

26. Hwang S, Jo M, Hong JE, Park CO, Lee CG, Yun M, et al. 2019. Zerumbone suppresses enterotoxigenic Bacteroides fragilis infectioninduced colonic inflammation through inhibition of NF-kappaBeta. Int. J. Mol. Sci. 20: 4560.

27. Hwang S, Lee CG, Jo M, Park CO, Gwon SY, Hwang S, et al. 2020. Enterotoxigenic Bacteroides fragilis infection exacerbates tumorigenesis in AOM/DSS mouse model. Int. J. Med. Sci. 17: 145-152.

28. Fadrosh DW, Ma B, Gajer P, Sengamalay N, Ott S, Brotman RM, et al. 2014. An improved dual-indexing approach for multiplexed 16S rRNA gene sequencing on the Illumina MiSeq platform. Microbiome 2: 6.

29. Bolger AM, Lohse M, Usadel B. 2014. Trimmomatic: a flexible trimmer for Illumina sequence data. Bioinformatics 30: $2114-2120$.

30. Masella AP, Bartram AK, Truszkowski JM, Brown DG, Neufeld JD. 2012. PANDAseq: paired-end assembler for illumina sequences. BMC Bioinformatics 13: 31

31. Eddy SR. 2011. Accelerated Profile HMM Searches. PLoS Comput. Biol. 7: e1002195.

32. Lee B, Moon T, Yoon S, Weissman T. 2017. DUDE-Seq: Fast, flexible, and robust denoising for targeted amplicon sequencing. PLoS One 12: $\mathrm{e} 0181463$.

33. Edgar RC. 2010. Search and clustering orders of magnitude faster than BLAST. Bioinformatics 26: 2460-2461.

34. Myers EW, Miller W. 1988. Optimal alignments in linear space. CABIOS 4: 11-17.

35. Fu L, Niu B, Zhu Z, Wu S, Li W. 2012. CD-HIT: accelerated for clustering the next-generation sequencing data. Bioinformatics 28: $3150-3152$

36. Segata N, Izard J, Waldron L, Gevers D, Miropolsky L, Garrett WS, et al. 2011. Metagenomic biomarker discovery and explanation. Genome Biol. 12: R60.

37. Yoon S-H, Ha S-M, Kwon S, Lim J, Kim Y, Seo H, et al. 2017. Introducing EzBioCloud: a taxonomically united database of 16S rRNA gene sequences and whole-genome assemblies. Int. J. Syst. Evol. Micr. 67: 1613.

38. Zackular JP, Baxter NT, Iverson KD, Sadler WD, Petrosino JF, Chen GY, et al. 2013. The gut microbiome modulates colon tumorigenesis. mBio 4: e00692-00613.

39. Sokol H, Seksik P, Rigottier-Gois L, Lay C, Lepage P, Podglajen I, et al. 2006. Specificities of the fecal microbiota in inflammatory bowel disease. Inflamm. Bowel. Dis. 12: 106-111.

40. Eckburg PB, Bik EM, Bernstein CN, Purdom E, Dethlefsen L, Sargent M, et al. 2005. Diversity of the human intestinal microbial flora. Science 308: 1635-1638.

41. Costello EK, Gordon JI, Secor SM, Knight R. 2010. Postprandial remodeling of the gut microbiota in Burmese pythons. ISME J. 4: $1375-1385$.

42. Jandhyala SM, Talukdar R, Subramanyam C, Vuyyuru H, Sasikala M, Nageshwar Reddy D. 2015. Role of the normal gut microbiota. World J. Gastroenterol. 21: 8787-8803.

43. Antunes EN, Ferreira EO, Falcao LS, Paula GR, Avelar KE, Barroso DE, et al. 2004. Non-toxigenic pattern II and III Bacteroides fragilis strains: coexistence in the same host. Res. Microbiol. 155: 522-524.

44. Chan JL, Wu S, Geis AL, Chan GV, Gomes TAM, Beck SE, et al. 2019. Non-toxigenic Bacteroides fragilis (NTBF) administration reduces bacteria-driven chronic colitis and tumor development independent of polysaccharide A. Mucosal. Immunol. 12: 164-177.

45. Hwang S, Jo M, Hong JE, Park CO, Lee CG, Rhee K-J. 2020. Protective effects of zerumbone on colonic tumorigenesis in Enterotoxigenic Bacteroides Fragilis (ETBF)-colonized AOM/DSS BALB/c Mice. Int. J. Med. Sci. 21: 857.

46. Hwang S, Jo M, Hong JE, Park CO, Lee CG, Yun M, et al. 2019. Zerumbone suppresses enterotoxigenic Bacteroides fragilis infection-

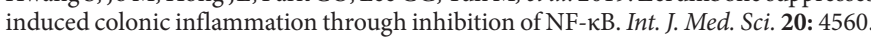

47. Gao Z, Guo B, Gao R, Zhu Q, Qin H. 2015. Microbiota disbiosis is associated with colorectal cancer. Front. Microbiol. 6: 20.

48. Corr SC, Li Y, Riedel CU, O'Toole PW, Hill C, Gahan CG. 2007. Bacteriocin production as a mechanism for the antiinfective activity of Lactobacillus salivarius UCC118. Proc. Natl. Acad. Sci. USA 104: 7617-7621.

49. Guinane CM, Lawton EM, O'Connor PM, O'Sullivan Ó, Hill C, Ross RP, et al. 2016. The bacteriocin bactofencin a subtly modulates gut microbial populations. Anaerobe 40: 41-49.

50. Kabeerdoss J, Jayakanthan P, Pugazhendhi S, Ramakrishna BS. 2015. Alterations of mucosal microbiota in the colon of patients with inflammatory bowel disease revealed by real time polymerase chain reaction amplification of $16 \mathrm{~S}$ ribosomal ribonucleic acid. Indian J. Med. Res. 142: 23-32.

51. Koliada A, Syzenko G, Moseiko V, Budovska L, Puchkov K, Perederiy V, et al. 2017. Association between body mass index and Firmicutes/Bacteroidetes ratio in an adult Ukrainian population. BMC Microbiol. 17: 120.

52. Ramakrishna BS. 2013. Role of the gut microbiota in human nutrition and metabolism. J. Gastroen. Hepatol. 28 Suppl 4: 9-17.

53. Mariat D, Firmesse O, Levenez F, Guimaraes V, Sokol H, Dore J, et al. 2009. The Firmicutes/Bacteroidetes ratio of the human microbiota changes with age. BMC Microbiol. 9: 123.

54. Kim H-R, Rhee K-J, Eom Y-B. 2019. Anti-biofilm and antimicrobial effects of zerumbone against Bacteroides fragilis. Anaerobe 57: 99-106. 